\title{
DIFFÉRENTES APPROCHES TRADUCTOLOGIQUES FACE AU PROBLÈME DE L'AMPLIFICATION EN TRADUCTION
}

\author{
Magdalena SzEFLIŃSKA-BARAN \\ Université de Łódź
}

\begin{abstract}
The phenomenon of the text amplification in the process of translation has been present both in translatology and in translation practice for a long time. It is often considered as one of the translation universals, that is the processes which accompany the translation of the text regardless of their typological characteristics (literary, journalistic or specialised texts) or translation's languages. In general, we can define the amplification as an expansion of the target text in its formal and signification layer comparing to the source text. The present study aims to show the possible methodological approaches to this phenomenon from the translatological point of view and to analyse sources and forms of the amplification in the literary translation.
\end{abstract}

L'inspiration du présent article vient aussi bien de l'observation du processus de traduction et de son produit obtenu comme texte cible que de la réflexion théorique exprimée entre autres par Antoine Berman (1999: 56) : « [...] l'allongement se produit - à des degrés divers - dans toutes les langues traduisantes, et il n'a pas essentiellement une base linguistique. [...] il s'agit d'une tendance inhérente au traduire en tant que tel $»$. La réflexion est centrée sur le problème de l'amplification du texte d'arrivée par rapport au texte de départ au cours de l'opération de traduction du français vers le polonais. Ce phénomène qui enferme différents procédés formels et sémantiques consiste, en termes très généraux, à augmenter le volume du texte cible par rapport à l'original. On a constaté à plusieurs reprises que le texte d'arrivée dépasse le cadre de l'original du point de vue soit quantitatif soit qualitatif. Ce phénomène, analysé jusqu'à présent surtout au niveau structural 
dans une paire de langues donnée, montre qu'il y a sûrement d'autres possibilités d'analyse nous procurant des réponses aux questions concernant les sources et les formes de l'amplification en traduction littéraire. Du point de vue théorique, il se situe au carrefour de différentes approches et méthodologies traductologiques : interprétative, cognitive, poétologique ou même structurale, qui nous permettent de l'interpréter soit comme une technique ou stratégie de traduction, soit comme une tendance universelle, endogène de la traduction comme processus ou même une figure de traduction. Notre objectif serait de trouver des traits définitoires de la notion d'amplification pour pouvoir la situer parmi d'autres phénomènes apparentés.

La définition d'amplification proposée par Hurtado Albir (2001 : 633-634) est la suivante :

[A]mpliación lingüística: Técnica de traducción que consiste en añadir elementos lingüísticos. Se opone a la técnica de compresión lingüística. La noción de amplificación : técnica de traducción que consiste en introducir precisiones no formuladas en el texto original: informaciones, paráfrasis explicativas. Incluye las notas del traductor. Se opone a la técnica de elisión.

Berman (1999), en soulignant que toute traduction est tendanciellement plus longue que l'original, définit ce phénomène nommé allongement comme étant un résultat de deux autres tendances déformantes, à savoir de la rationalisation (portant sur les structures syntaxiques de l'original en les recomposant selon une certaine idée de l'ordre linéaire d'un discours) et de la clarification (qui concerne le niveau de clarté sensible des mots ou leur sens et qui tend à imposer du défini là où l'original s'exprime par l'indéfini). La clarification est inhérente à la traduction, dans la mesure où tout acte de traduire est explicitant. L'explicitation peut être la manifestation de quelque chose qui n'est pas apparent, mais réprimé dans l'original et que la traduction met au jour. Mais en un sens négatif, l'explication vise à rendre clair ce qui ne l'est pas et ne veut pas l'être dans l'original (le passage de la polysémie à la monosémie, la traduction paraphrasante ou explicative sont des modes de clarification). Berman distingue deux types d'allongement :

- un allongement vide, un simple ajout qui n'apporte rien au texte et qui a un caractère typiquement quantitatif (nommé aussi horizontal ${ }^{1}$ ) ;

- un allongement qui augmente la parlance ou la signifiance d'un texte (la surtraduction ou l'amplification verticale ${ }^{2}$ ).

\footnotetext{
${ }^{1}$ Szeflińska (2003).

${ }^{2}$ Ibid.
} 
Nida (1964) traite l'explicitation sous la rubrique de l'ajout au texte cible, dont il définit plusieurs types, par exemple : expliciter une expression elliptique, ajouter une information grammaticale, amplifier un énoncé laconique. Chez Nida, la technique de l'amplification est celle qui illustre le mieux l'explicitation : elle consiste à ajouter des informations inexistantes dans l'original lorsque des éléments sémantiques importants et implicites de la langue source nécessitent une identification explicite dans la langue du récepteur.

Edward Balcerzan (1968/1998) énumère l'amplification (à côté de la réduction, l'inversion et la substitution) parmi quatre types de transformations ayant lieu pendant l'opération de traduction. D'ailleurs les transformations citées ci-dessus viennent de la rhétorique classique. Selon ce théoricien polonais, l'amplification consiste à ajouter au texte cible des éléments nouveaux, sous-jacents, cachés dans l'implicite de l'original. L'amplification concerne plutôt la tonalité de l'original que son contenu conceptuel. Elle se manifeste également comme procédé compensatoire face à la sous-traduction dans des textes littéraires dont le style se caractérise par la simplicité syntaxique ou stylistique.

La notion d'amplification est également connue dans les études littéraires comme une des figures de style, un procédé littéraire, fondé entre autres sur une gradation entre les termes d'une énumération ou dans la construction d'un paragraphe dans le but de renforcer le propos. Amplifier, c'est développer les idées par le style pour leur donner plus d'ornement, plus d'étendue ou plus de force. En rhétorique, l'amplification est utilisée pour faire ressortir l'importance du sujet par exemple dans le discours démonstratif.

L'objet de cette étude est entre autres de dénoncer l'inanité de la terminologie utilisée en traductologie pour décrire le phénomène d'amplification. Les procédés de traduction participant à l'amplification du texte source, tels qu'ils ont été exposés par Vinay et Darbelnet dans leur Stylistique comparée (1958), méritent une critique, vu d'abord la terminologie utilisée, puis le type de description de la traduction elle-même. La terminologie de Vinay et Darbelnet souffre de deux maux : un nombre très bas de termes pour décrire une opération si complexe et l'inadéquation de termes mal définis ou mal intégrés à une théorie de la traduction qui soit satisfaisante. Ce qui se cache derrière cette typologie, c'est une vision erronée de la traduction et par là même de sa théorie. La notion de base pour pouvoir parler d'amplification est celle d'unité de traduction. Elle ne peut pas s'identifier dans le texte source sans prendre en considération le texte cible, la phase d'interprétation du texte de départ et le processus de conceptualisation $\mathrm{du}$ sens à transmettre pendant l'opération de traduction. L'échec des essais de l'analyse de l'amplification au sein de la traductologie découle, entre autres, des approches inappropriées appliquées aux études sur l'amplification. La démarche 
structurale et comparative laisse beaucoup à désirer. L'essentiel de la théorie de la traduction devrait être fondé sur l'observation de l'action du traducteur, qui est un mélange d'herméneutique, de production d'équivalence et de réécriture. Dans le présent article, nous ne voulons nous référer ni directement à la classification des procédés de traduction instituée par Vinay et Darbelnet (1966 : 55), ni à sa critique faite par Michel Ballard (2006), mais nous voudrions proposer une démarche analytique de l'amplification dans le cadre de la théorie de la traduction interprétative.

Les notions apparentées telles que : l'étoffement, le foisonnement, la dilution et l'amplification, la surtraduction chez Vinay et Darbelnet, découlent de l'approche linguistique comparative de la traduction et consistent dans une analyse quantitative du nombre d'unités lexicales, confondues d'ailleurs avec des unités de traduction, dans l'original et le texte d'arrivée. Le foisonnement est également défini par Durieux (1990) comme une surtraduction, un ajout par le traducteur des syntagmes apparemment explicatifs ou porteurs d'informations complémentaires. Elle affirme que tous les textes foisonnent indépendamment des langues en présence pendant le processus de traduction et que ce foisonnement est attribuable au manque de méthode du traducteur, il s'agit de la traduction spécialisée. Le foisonnement a surtout été un objet de recherche statistique (le coefficient de foisonnement) dans le domaine de la traduction de textes de spécialité s'appuyant sur les études de corpus. La typologie des procédés de traduction présente son intérêt dans l'orientation vers le processus de traduction qui enferme les composants tels que : le traducteur, l'interprétation herméneutique de l'original.

La notion de procédés de traduction a été introduite par Vinay et Darbelnet en 1958, dans la Stylistique comparée du français et de l'anglais. Cet ouvrage, qui se donne ouvertement comme une Méthode de traduction (sous-titre utilisé par les auteurs eux-mêmes), peut être considéré comme une production caractéristique de la linguistique appliquée à la traduction avec des visées didactiques et pratiques. La notion de procédés est étroitement associée à cette démarche, puisqu'ils proposent d' " étudier sur des exemples aussi précis et aussi probants que possible les mécanismes de la traduction, en dériver des procédés, et pardelà les procédés retrouver les attitudes mentales, sociales, culturelles qui les informent » (1966:26). En plusieurs endroits de leur manuel, Vinay et Darbelnet utilisent des termes tels que amplification, économie, dilution, concentration, étoffement, dépouillement, qui se recoupent et se chevauchent de façon assez anarchique. Si l'on considère la définition de l'étoffement, ce phénomène est décrit comme étant « une variété de l'amplification appliquée aux prépositions françaises qui ont besoin d'être étoffées » (ibid., p. 12). Par ailleurs, on notera 
que la définition de l'amplification (« cas où la LA emploie plus de mots que la LD pour exprimer la même idée » (ibid., p. 5) manque de clarté. Elle reprend en termes non linguistiques la définition de la dilution : « répartition d'un signifié sur plusieurs signifiants » (ibid., p. 7). Encore une fois, la formulation, pour l'amplification, n'est pas bonne, elle est le reflet d'une absence d'élaboration de la théorie. L'étoffement est défini, de façon assez générale, comme « le renforcement d'un mot qui ne suffit pas à lui-même et qui a besoin d'être épaulé par d'autres » (ibid., p. 109). Alors que dans le glossaire, l'étoffement était « une variété d'amplification appliquée aux prépositions françaises qui ont besoin d'être étoffées par l'adjonction d'un adjectif ou d'un participe passé ou même d'un nom, alors que les prépositions anglaises se suffisent à elles-mêmes » (ibid., p. 9).

Il est surtout clair que Vinay et Darbelnet ont esquissé une description d'un phénomène majeur en traduction, une catégorie nommée "la différence de concentration » (Ballard, 1994) qui regroupe un certain nombre de phénomènes tels que l'étoffement et l'effacement, le développement et la réduction. Or cette catégorie n'apparaît pas dans le tableau général de leurs procédés parce que celui-ci ne part pas d'une vision globale de la traduction et que leurs procédés ne sont pas rattachés de façon synthétique au processus de traduction. Certains viennent chercher dans la traductologie ou dans la linguistique contrastive des techniques infaillibles qui permettraient de réussir la traduction. Nous préférons parler d'opérations qui reflètent la triple démarche de la traduction, à savoir : des opérations d'interprétation, de paraphrase et de réexpression. L'observation de la traduction devrait nous permettre de mieux accéder à la connaissance de la compétence du traducteur et donc à une meilleure acquisition, une acquisition facilitée ou plus éclairée, de cette compétence ; elle ne saurait cependant se substituer à l'intelligence, au talent, à une compétence innée, et surtout à la motivation. La traductologie ne peut sécréter une machine qui dispenserait de penser et d'agir, pas plus que la linguistique ou la textologie ; ces sciences permettent une meilleure conceptualisation des problèmes, de meilleures analyses, des prises de décision plus conscientes.

Une des failles de l'ouvrage de Vinay et Darbelnet est que les procédés non seulement sont en nombre trop petit pour rendre compte de l'opération, mais aussi qu'ils ne sont pas assez explicitement rapportés à l'opération elle-même ainsi qu'à l'outil conceptuel permettant de pouvoir servir de cadre à son analyse et à sa conceptualisation : l'unité de traduction.

Ce genre de traduction est bien la preuve qu'on ne peut pas prédécouper des unités de traduction dans le texte de départ. C'est par rapport à la reformulation, autour de l'équivalence, tout autant que de la réécriture, que se construit l'unité 
de traduction. La traduction est un processus complexe que l'on peut ramener à un modèle triadique, dont le cœur est une opération de paraphrase synonymique visant à produire un texte équivalent au texte de départ. Cette position centrale de l'équivalence a amené nombre de pédagogues, de théoriciens, et même de linguistes, à axer l'étude de la traduction sur la recherche de procédés, oubliant d'une part qu'il n'y a pas de recettes et, d'autre part, que la traduction comporte au moins deux autres phases tout aussi importantes : la phase herméneutique et la phase de réécriture.

L'unité de traduction est un élément constituant d'un tout qui a sa source, ou base formelle, dans le texte de départ, son aboutissement dans le texte d'arrivée, et qui passe pour sa réalisation par le cerveau du traducteur; il s'agit donc d'un ensemble à configuration variable selon l'individu qui le construit ; ce qui signifie qu'il faut intégrer la subjectivité dans l'unité de traduction. À partir de là, on peut dire que l'objet étant le texte, il y a constitution d'une unité de travail en traduction lorsque le traducteur, après interprétation des formes, met en rapport une unité constituante du texte de départ avec le système de la langue d'arrivée en vue de produire une équivalence acceptable, susceptible de contribuer à la réécriture d'un texte dont l'équivalence globale par rapport au texte de départ doit s'accommoder d'ajustements internes dictés par sa cohérence et sa lisibilité. Cela signifie que sur le plan formel, il existe plusieurs types d'unités de traduction selon que leur base apparente est dans le texte de départ (et c'est le plus souvent le cas) ou plutôt générée par la constitution du texte d'arrivée et les exigences extralinguistiques de la culture d'accueil.

L'approche herméneutique et processuelle de la question d'amplification dans le cadre de la théorie interprétative, appelée aussi la théorie du sens, permet de voir toute la complexité de ce phénomène en traduction. Les observations faites dans les textes traduits permettent de constater que l'essentiel du problème en question ne réside pas dans l'allongement du texte ou autrement dit dans des changements d'ordre quantitatif, mais dans une augmentation ou renforcement $\mathrm{du}$ volume conceptuel, affectif et stylistique du texte d'arrivée par rapport à l'original. Le point de départ du processus qui aboutit à une amplification en traduction a sa source dans l'interprétation du texte par le traducteur et il peut se faire accompagner par une forme de foisonnement formel. L'opération de traduction engage principalement deux sphères d'activité du traducteur : la première correspond à la lecture interprétative de l'original laquelle a pour objectif de saisir les unités du sens, la deuxième se réalise dans la réécriture, la réexpression des unités de traduction en langue d'arrivée, donc création d'un texte nouveau. 
Nous voudrions préciser ce que nous entendons par l'amplification :

- les cas où la LA emploie plus de mots que la LD pour exprimer la même idée (Vinay et Darbelnet, op. cit., p. 5) ;

- la notion d'amplificación : técnica de traducción que consiste en introducir precisiones no formuladas en el texto original: informaciones, paráfrasis explicativas. Incluye las notas del traductor. Se opone a la técnica de elisión. Economía (Hurtado Albir, op. cit., p. 633-634);

- l'amplification consiste à ajouter au texte cible des éléments nouveaux, sous-jacents, cachés dans l'implicite de l'original (Balcerzan, 1968/1998).

Il est donc clair que l'amplification ne réside pas dans une simple augmentation du nombre d'éléments structuraux constituant le texte d'arrivée par rapport au texte de départ. Elle peut être conçue comme un changement voulu, introduit par le traducteur, qui consisterait en chaque surcroît d'informations dans le message traduit ayant lieu pendant la traduction, celui-là n'étant pas dû aux changements nécessaires provoqués par les structures de deux langues en contact. Dans le cadre de l'approche interprétative, nous pouvons distinguer deux types majeurs de l'amplification :

1) amplification qualitative ou verticale - peut être conçue comme un changement voulu, introduit par le traducteur, qui consisterait en chaque surcroît d'informations verbalisées dans le message traduit, qui consiste soit à verbaliser les informations implicites, contenues dans le texte de départ (ce type de procédé est libre quant à son existence car il dépend du choix du traducteur ; par contre il est déterminé quant à son contenu par la totalité d'informations implicites et explicites dans l'original. Cette modification est appelée par certains auteurs 《explicitation ») ; soit à ajouter des éléments absents de l'original ; elle élargit le sens du message de départ. Ces ajouts s'avèrent justifiés par le processus de compréhension et d'interprétation du sens par le traducteur qui construit sa propre vision du texte source et qui dispose de ses connaissances préétablies déterminant sa manière de voir, de comprendre le message et de le réexprimer ;

2) amplification quantitative ou horizontale - une simple augmentation du nombre d'éléments structuraux constituant le texte d'arrivée par rapport au texte de départ. L'amplification horizontale :

- concerne les différences au niveau structural de deux langues en contact : au niveau des correspondances grammaticales, lexicales ou syntaxiques (selon Lederer, 1984);

- a un caractère quantitatif (le nombre d'unités de langue augmente en TA par rapport au TD) ; 
- enferme différents procédés tels que : étoffement, dilution, ampliación lingüistica ;

- a sa source dans la structure de la langue d'arrivée face à celle de la LD, dans la phase de réexpression en LA.

On ne peut pas nier que la traduction d'un texte fasse appel, assez souvent, à une explicitation des informations implicites et qu'elle repose sur un processus d'exégèse des données extralinguistiques contenues dans le texte, ce qui donne lieu à la situation où on a la même quantité d'information mais la distribution entre l'explicite et l'implicite est différente. Dans la plupart des cas, cette explicitation est l'une des raisons pour lesquelles le texte traduit devient plus ample, plus long par rapport au texte de départ. Cette situation résulte très souvent du dynamisme de l'opération de traduction, des va-et-vient entre le texte et son lecteur - le traducteur. L'analyse de l'amplification et de différents procédés qui la soustendent montre que l'explicitation joue un rôle primordial dans l'amplification qui a lieu pendant la traduction. Nous espérons avoir réussi à montrer que la notion de procédé est inadéquate pour rendre compte de l'amplification, en raison de sa pauvreté conceptuelle et théorique. Nous proposons de lui substituer une analyse du travail réel ou supposé du traducteur dans le cadre d'unités de traductions dont les composantes formelles visibles ou invisibles dans le TD et le TA sont unies par une relation d'équivalence.

Enfin, ce qui nous semble devoir caractériser la traductologie, c'est son acceptation d'un inévitable degré d'imprévisibilité de la traduction qui se manifestera par une plus grande modalisation d'unéventuel système d'équivalences auquel on pourrait aboutir par l'observation de corpus. Et surtout, ce système est inéluctablement flanqué ou adossé à l'exploration de deux composantes essentielles : l'herméneutique du texte de départ et la constitution du texte d'arrivée. L'objet de la traductologie n'est pas la langue, même si on peut profiter de la traduction pour étudier ou affiner sa perception des langues (et ceci est manifeste dans l'usage qui est fait de la traduction dans le cadre de l'enseignement de langues) ; l'objet de la traductologie n'est pas le langage mais la traduction, c'est-à-dire une activité de paraphrase synonymique interlinguistique dont la base est un texte, matière à interprétation, et dont l'objet est la reconstitution, donc l'écriture d'un texte équivalent pour un public d'une autre culture. La traductologie est une science d'observation intégrant des paramètres qui permettent de dessiner des repères fondamentaux indicatifs tels que les spécificités linguistiques et les caractéristiques des textes à traiter en tant que genres, mais une science qui intègre également des facteurs qui introduisent des variables telles que les conditions sociolinguistiques de production de la traduction, la subjectivité du traducteur au 
niveau de l'interprétation, des choix et des options de traduction, tout autant que le paramètre de la créativité. La notion d'amplification analysée dans la perspective herméneutique s'avère propre à rendre compte de la complexité de l'opération de traduction et se situe clairement du côté des universaux traductologiques en dépassant le cadre de son analyse jusqu'à présent le plus souvent mentionné, à savoir celui des procédés de traduction.

\section{Références bibliographiques}

BALCERZAN, Edward (1968/1998), Literatura z literatury. Strategie tlumaczy, Katowice, Śląsk.

BALLARD, Michel (1991), Éléments pour une didactique de la traduction, Thèse de doctorat, Université Sorbonne nouvelle - Paris 3.

BALLARD, Michel (1992), Le Commentaire de traduction anglaise, Paris, Nathan.

BALLARD, Michel (1994 [1987]), La Traduction de l'anglais au français, 2éd. revue et corrigée, Paris, Nathan.

BALLARD, Michel (2001), Le Nom propre en traduction, Paris, Ophrys.

BALLARD, Michel (2006), " À propos des procédés de traduction », Palimpsestes. Revue de traduction, $\mathrm{n}^{\circ}$ Hors Série, pp. 113-130.

BERMAN, Antoine (1999 [1985]), La traduction et la lettre ou l'Auberge du lointain, Paris, Éditions du Seuil.

CHUQUET, Hélène, PAILLARD, Michel (1987), Approche linguistique des problèmes de traduction, Paris, Ophrys.

COCHRANE, Guylaine (1995), "Le foisonnement, phénomène complexe », TTR : traduction, terminologie, rédaction, vol. 8, $\mathrm{n}^{\circ}$ 2, pp. 175-193.

DELISLE, Jean (1993), La Traduction raisonnée, Ottawa, Les Presses de l'Université d'Ottawa (coll. « Pédagogie de la traduction »).

DURIEUX, Christine (1990), « Le foisonnement en traduction technique d'anglais en français », Meta, vol. XXXV, n 1, pp. 55-60.

GRELLET, Françoise (1991), Apprendre à traduire. Typologie d'exercices de traduction, Nancy, Presses Universitaires de Nancy.

HARDIN, Gérard, PICOT, Cynthia (1990), Translate. Initiation à la pratique de la traduction, Paris, Dunod.

HURTADO ALBIR, Amparo (2001), Traducción y Traductología. Introducción a la Traductología, Madrid, Ediciones Cátedra Lingüística.

LADMIRAL, Jean-René (1979), Traduire : théorèmes pour la traduction, Paris, Payot.

LEDERER, Marianne (1984), «Implicite et explicite», in Interpréter pour traduire (D. Seleskovitch, M. Lederer éds), Paris, Didier Érudition.

NIDA, Eugene (1964), Toward a Science of Translating, Leiden, E. J. Brill.

SZEFLIŃSKA, Magdalena (2003), Sources et formes d'amplification dans les traductions littéraires du français en polonais, Łódź/Lask, Oficyna Wydawnicza LEKSEM.

VAN HOOF, Henri (1989), Traduire l'anglais. Théorie et pratique, Louvain-la-Neuve, Duculot. 
VINAY, Jean-Paul, DARBELNET, Jean (1966 [1958]), Stylistique comparée du français et de l'anglais, Paris, Didier.

VINAY, Jean-Paul, DARBELNET, Jean (1995), Comparative Stylistics of French and English, trad. J. C. Sager et M.-J. Hamel, Amsterdam/Philadelphia, John Benjamins.

VRECK, Françoise (1992), ABC de la version anglaise, Paris, Longman France (coll. « Longman Université $»)$. 